\title{
Pre-impact monitoring of Comet 9P/Tempel 1, the Deep Impact target
}

\author{
L. M. Lara ${ }^{1}$, H. Boehnhardt ${ }^{2}$, R. Gredel ${ }^{3}$, P. J. Gutiérrez ${ }^{1}$, J. L. Ortiz ${ }^{1}$, R. Rodrigo ${ }^{1}$, and M. J. Vidal-Nuñez ${ }^{1}$ \\ 1 Instituto de Astrofísica de Andalucía, CSIC, PO Box 3004, 18080 Granada, Spain \\ e-mail: lara@iaa.es \\ 2 Max-Planck Institut für Sonnensystemforschung, Max-Planck-Str. 237191 Katlenburg-Lindau, Germany \\ e-mail: boehnhardt@mps.mpg.de \\ 3 Max-Planck Institut für Astronomie, Königstuhl 17, 69117 Heidelberg, Germany \\ e-mail: gredel@caha.es
}

Received 15 July 2005 / Accepted 14 September 2005

\section{ABSTRACT}

Comet 9P/Tempel 1, the target of the Deep Impact Mission, has been monitored for 6 months aiming at its characterization before the impact experiment. During this period of time, the coma has gone through a slow morphological evolution from a wide structure in the south-western quadrant in mid-February to a porcupine pattern in mid-April and up to seven features identified in June. In addition to this evolution, an arclet in the western coma hemisphere was detected on June 14, related to an outburst event. Interpretation of these features and their evolution seems to indicate the presence of at least 3 or 4 very active regions on the nucleus, consistent with the rotation axis being oriented close to previous solutions found by Belton et al. (2005), or close to the angular momentum vector of the orbital motion of the comet. The value of $A f \rho$ varies with heliocentric distance as $r_{\mathrm{h}}^{-6.71}$; slightly enhanced $A f \rho$ (above the $r_{\mathrm{h}}^{-6.71}$ curve) was observed from mid-February until the end of March, when fan-shaped structures appeared in the coma for the first time. Somewhere between mid-April to mid-May (i.e. 80 to 60 days before perihelion), the comet peaked in activity. In terms of gas production rates, $\mathrm{CN}, \mathrm{C}_{2}$ and $\mathrm{C}_{3}$ have been obtained at $r_{\mathrm{h}} \sim 1.7,1.60$ and $1.51 \mathrm{AU}$, being slightly below those derived from previous passages. Abundance ratios of these species indicate that comet 9P/Tempel 1 is classified as typical in terms of $\mathrm{C}_{2}$ abundance. The surface brightness profiles of the continuum, either azimuthally averaged profiles from the broadband images or in north-south direction from the long-slit spectra can be well fit with $-1.9 \leq m \leq-1.14$ in $\log B-\log \rho$ representation. Steeper slopes are obtained at larger $r_{\mathrm{h}}$ which might be related to variable dust size distribution with distance from the nucleus due to the radiation pressure dynamics and/or physical processing of the dust grains (sublimation, fragmentation). Normalized color of the dust inside the coma in the north-south direction is measured to be $\sim 20-30 \% / 100 \mathrm{~nm}$.

Key words. comets: individual: 9P/Tempel 1

\section{Introduction}

In January 2005 NASA's Deep Impact (DI) spacecraft was launched to perform a cratering experiment at Comet 9P/Tempel 1 on 4 July 2005: a $360 \mathrm{~kg}$ impactor hit the cometary nucleus at $10.2 \mathrm{~km} \mathrm{~s}^{-1}$ speed to excavate a crater and to initiate new activity of the nucleus. The scientific goals of this unique experiment are - apart from studying crater physics - to characterize the target, $9 \mathrm{P} /$ Tempel 1 , as a cometary nucleus, i.e. as a representative of the primordial bodies from the formation period of the planetary system. Due to the very limited instruments on board the fly-by spacecraft (two cameras and a spectrometer) that can follow the impact for $800 \mathrm{~s}$, a significant science contribution is expected from Earth-based observations. The published measurements on the gas and dust production of the comet (for instance Osip et al. 1992; Cochran et al. 1992; Storrs et al. 1992) were obtained in the last two decades of the last century, i.e. more than two orbital revolutions of the comet ago. In preparation of the DI mission, more recent results on the comet (for instance nucleus parameters like size, rotation), collected during the past perihelion-to-aphelion arc of the orbit, are published by Meech et al. (2005) and Belton et al. (2005).

Here, we present imaging and spectroscopy observations of 9P/Tempel 1 collected during a pre-impact monitoring campaign between early January 2005 and 18 June 2005 (18 days before impact). The goal of this program was to study the gas and dust production and the coma morphology for about half a year up to the encounter in order to provide references on the pre-impact state of the comet.

\section{Observations and data reduction}

Imaging: Comet 9P/Tempel 1 was monitored from the Calar Alto Observatory (Consejo Superior de Investigaciones Científicas-Max Planck Gesellschaft, CSIC-MPG, near Almeria, Spain) and Sierra Nevada Observatory (CSIC, 
Table 1. Observation Logs and results.

\begin{tabular}{|c|c|c|c|c|c|c|c|c|c|c|c|c|}
\hline \multirow{2}{*}{$\begin{array}{l}\text { Date } \\
2005\end{array}$} & \multirow[t]{2}{*}{ UT } & \multirow{2}{*}{$\begin{array}{c}r_{\mathrm{h}} \\
(\mathrm{AU})\end{array}$} & \multirow{2}{*}{$\begin{array}{c}\Delta \\
(\mathrm{AU})\end{array}$} & \multirow[t]{2}{*}{ PA } & \multirow{2}{*}{$\begin{array}{l}A f \rho \\
(\mathrm{cm})\end{array}$} & \multirow[t]{2}{*}{$m$} & \multicolumn{3}{|c|}{$Q\left(\mathrm{~s}^{-1}\right)$} & \multicolumn{3}{|c|}{$\log Q^{\prime} \mathrm{s}$} \\
\hline & & & & & & & $\mathrm{CN}$ & $\mathrm{C}_{2}$ & $\mathrm{C}_{3}$ & $\mathrm{C}_{2} / \mathrm{CN}$ & $\mathrm{C}_{3} / \mathrm{CN}$ & Af $\rho / \mathrm{CN}$ \\
\hline Jan. 12 & $05: 56-06: 26$ & 2.21 & 1.75 & 290.2 & 55.8 & -1.72 & & & & & & \\
\hline Jan. 17 & $02: 59-03: 33$ & 2.18 & 1.67 & 289.0 & 58.7 & -1.70 & & & & & & \\
\hline Jan. 20 & $04: 30-04: 58$ & 2.16 & 1.62 & 288.3 & 53.0 & -1.91 & & & & & & \\
\hline Jan. 31 & $04: 43-05: 22$ & 2.09 & 1.45 & 285.0 & 76.8 & -1.70 & - & - & - & & & \\
\hline Feb. 02 & $04: 27-06: 00$ & 2.09 & 1.42 & 284.3 & 75.4 & -1.72 & & & & & & \\
\hline Feb. 03 & $05: 39-06: 13$ & 2.07 & 1.39 & 283.6 & 77.2 & -1.32 & & & & & & \\
\hline Feb. 04 & $03: 42-04: 22$ & 2.08 & 1.39 & 283.6 & 79.5 & -1.66 & & & & & & \\
\hline Feb. 17 & $04: 17-04: 46$ & 2.00 & 1.21 & 277.7 & 96.1 & -1.74 & & & & & & \\
\hline Feb. 20 & $05: 45-06: 07$ & 1.98 & 1.17 & 275.9 & 116.3 & -1.69 & & & & & & \\
\hline Mar. 06 & $01: 38-02: 10$ & 1.91 & 1.01 & 264.7 & 157.7 & -1.56 & & & & & & \\
\hline Mar. 13 & $03: 13-04: 49$ & 1.87 & 0.94 & 255.9 & 159.4 & -1.69 & & & & & & \\
\hline Mar. 27 & $00: 08-01: 18$ & 1.80 & 0.83 & 226.0 & 242.2 & -1.57 & - & - & - & & & \\
\hline Apr. 12 & Full night & 1.72 & 0.75 & 158.1 & 268.5 & -1.42 & - & - & - & & & \\
\hline Apr. 13 & Full night & 1.72 & 0.74 & 157.6 & 277.7 & -1.41 & $1.10 \times 10^{24}$ & $1.07 \times 10^{24}$ & $1.27 \times 10^{23}$ & -0.01 & -0.94 & -21.59 \\
\hline Apr. 14 & Full night & 1.71 & 0.74 & 157.1 & 287.7 & -1.43 & $1.26 \times 10^{24}$ & $1.72 \times 10^{24}$ & $1.47 \times 10^{23}$ & 0.14 & -0.93 & -21.64 \\
\hline Apr. 15 & Full night & 1.71 & 0.74 & 156.5 & 272.9 & -1.39 & $2.15 \times 10^{24}$ & $2.59 \times 10^{24}$ & $2.01 \times 10^{23}$ & 0.08 & -1.03 & -21.90 \\
\hline May 11 & $20: 39-21: 14$ & 1.60 & 0.72 & 127.9 & 248.1 & -1.42 & $3.31 \times 10^{24}$ & $2.64 \times 10^{24}$ & $3.02 \times 10^{23}$ & -0.10 & -1.04 & -22.12 \\
\hline May 14 & $21: 12-21: 32$ & 1.59 & 0.72 & 125.9 & 210.9 & -1.12 & & & & & & \\
\hline May 18 & $20: 39-21: 14$ & 1.58 & 0.73 & 123.5 & 219.5 & -1.39 & & & & & & \\
\hline May 22 & $20: 39-21: 14$ & 1.57 & 0.73 & 121.6 & 214.3 & -1.38 & & & & & & \\
\hline May 25 & $21: 08-22: 36$ & 1.56 & 0.74 & 120.3 & 205.3 & -1.17 & & & & & & \\
\hline May 26 & $20: 59-21: 24$ & 1.56 & 0.74 & 119.9 & 202.3 & -1.19 & & & & & & \\
\hline Jun. 02 & $23: 52-00: 12$ & 1.54 & 0.76 & 117.7 & 186.8 & -1.18 & & & & & & \\
\hline Jun. 03 & $20: 41-21: 14$ & 1.54 & 0.76 & 117.2 & 156.7 & -1.15 & & & & & & \\
\hline Jun. 05 & $20: 59-22: 32$ & 1.53 & 0.77 & 116.6 & 211.7 & -1.18 & & & & & & \\
\hline Jun. 06 & $20: 30-23.50$ & 1.53 & 0.78 & 116.3 & 172.7 & -1.19 & & & & & & \\
\hline Jun. 08 & $21: 04-21: 30$ & 1.53 & 0.78 & 115.8 & 210.5 & -1.19 & & & & & & \\
\hline Jun. 11 & $21: 00-21: 20$ & 1.52 & 0.80 & 115.1 & 135.42 & -1.23 & & & & & & \\
\hline Jun. 18 & $20: 44-21: 15$ & 1.51 & 0.82 & 113.7 & 164.7 & -1.15 & $1.90 \times 10^{24}$ & $2.11 \times 10^{24}$ & $2.17 \times 10^{23}$ & 0.04 & -0.94 & -22.06 \\
\hline
\end{tabular}

PA refers to the position angle of the projected extended Sun-target radius vector, counted North over East. $r_{\mathrm{h}}$ and $\Delta$ are the heliocentric and geocentric distances of the comet during our observations, respectively. $m$ : slope of the linear fit $\log B$ vs. $\log \rho$. Standard deviation of these fits is always lower than $1 \%$.

Af $\rho$ values are given for a circular aperture of $5000 \mathrm{~km}$ at the comet distance.

Uncertainties in $A f \rho$ and $Q$ 's are estimated to be $\leq 10 \%$ and $\leq 25 \%$ respectively, from the star calibration and sky subtraction.

near Granada, Spain) since January 2005. We used three different imaging systems for the observations: CAFOS $\left(2048 \times 2048\right.$ pixels, pixel size: $0{ }^{\prime} .53$, FOV $\left.18^{\prime} \times 18^{\prime}\right)$ and BUSCA $\left(4096 \times 4096\right.$ pixels, pixel size: 0 .' $^{\prime} 17$, FOV $11.6 \times 11.6)$ mounted at the $2.2 \mathrm{~m}$ telescope at Calar Alto, and Versarray CCD $(2048 \times 2048$ pixels, pixel size: 0.232 , FOV 7 '.9 $\times 7$ '.9) mounted on the $1.5 \mathrm{~m}$ telescope at the Sierra Nevada Observatory. For CAFOS, only the $1 \mathrm{x} 1 \mathrm{k}$ central pixels were used, whereas in the case of BUSCA and Versarray CCD a binning $2 \times 2$ was applied during read-out. Thus, the FOV covered by our observations is $9^{\prime} \times 9^{\prime}, 5{ }^{\prime} .8 \times 5^{\prime} .8$ and $7.9 \times 7$ 7 $^{\prime} 9$ for the three instruments mentioned above, respectively. The comet was imaged with Johnson $R$ (CAFOS) or $I$ (BUSCA) broadband filter typically every 3-5 days from Calar Alto
Observatory and with Johnson $R$ and $I$ filters during selected periods from the Sierra Nevada Observatory. Additionally, a 5 nights run at Calar Alto used Johnson BVRI and cometary $\mathrm{CN}$ in April 2004 (10th to the 14th). In each filter, consecutive series of 5 to 10 images were acquired. Appropriate bias and flat field frames were also taken each night. If photometric conditions prevailed, photometric standard stars were observed at airmass similar to the comet observations. Table 1 contains the observation logs for the photometric nights.

Spectroscopy: the spectroscopic measurements were done once every month using CAFOS with grism B200 and B400 (see http://www. caha.es/alises/cafos/cafos 22 .pdf for more details; observable spectral range between 3200 and $8800 \AA$ with a wavelength scale of $4.75 \AA$ per pixel, and 


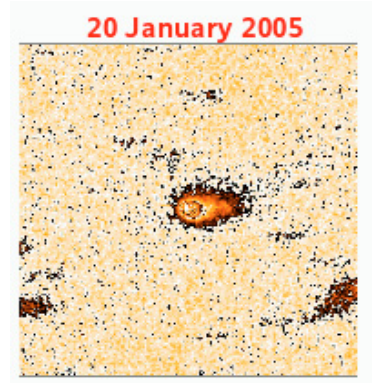

13 April 2005

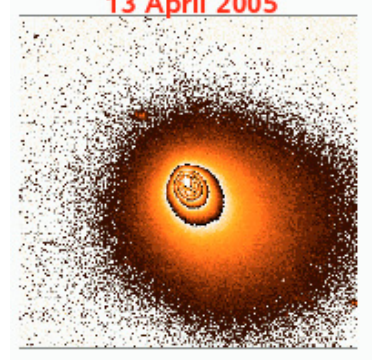

20 February 2005

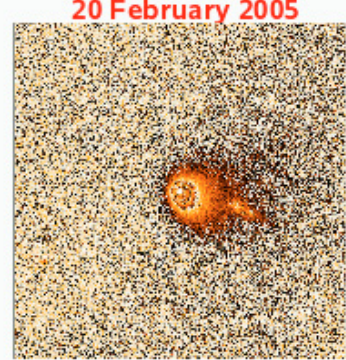

14 May 2005

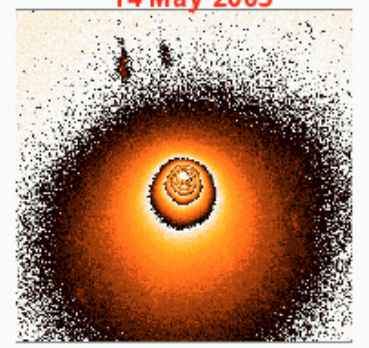

13 March 2005

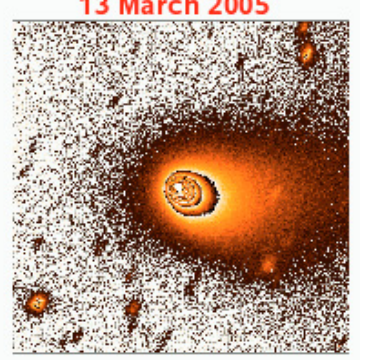

5 June 2005

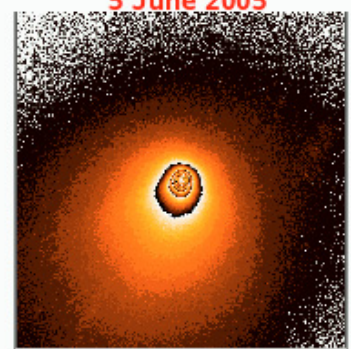

Fig. 1. Isophote images of Comet 9P/Tempel 1. The sequence shows the evolution of the dust coma from January to mid June 2005 (dates are given in the figure). The isophotes are displayed as envelopes of similar colour through a multiple jigsaw intensity transfer table. The individual images consist of a series of co-aligned and co-added exposures taken during a short time interval (typically 20 min). Background objects appear as trails due to the telescope tracking of the comet. North is up and East to the left. The field of view is $2.25 \times 2.25$ arcmin. The brightness peak in the coma (equivalent to the nucleus position) is placed in the center of the field of view.

between $2800 \AA$ and $1.0 \mu \mathrm{m}$ with a wavelength scale of $9.7 \AA$ per pixel, respectively). The slit of the spectrograph was orientated in north-south direction, giving dust and gas profiles at different cross-cuts through the coma, depending on the comet position in the sky. For absolute calibration, observations of appropriate spectrophotometric standard stars were acquired. All comet observations were done with telescope tracking at the comet's proper motion.

With the exception of the 5 night run in April 2005, all observations were done in service mode at both observatories. Details on the image and spectral reduction and calibration can be found in Lara et al. (2001, 2004a,b) and they are not repeated here. Since the gas emission of 9P/Tempel 1 does not cover the entire length of the slit, it was possible to extract the sky contamination directly from the edges of the frame.

\section{Data analysis and results}

\subsection{Coma morphology}

For the enhancement of morphological structures in the coma, calibrated $R$ and $I$ filter images (for examples see Fig. 1) are processed by two methods: (1) Adaptive Laplace filtering (Boehnhardt \& Birkle 1994 and references therein) applied to all images and (2) radial renormalization (for instance A'Hearn et al. 1986) used for verification of the strongest features in selected high signal-to-noise ratio (SNR) exposures of our dataset. Figure 2 shows the Laplace filtered version of the images from Fig. 1. The sequence depicts the evolution of coma structures in the comet between early January 2005 until shortly before impact. In Fig. 3 a direct comparison of the enhancement results (see sub-frames for 14 June 2005) for the Laplace filtering and renormalization technique is presented.
While the Laplace filtered images show coma structures in greater detail, the renormalized images are useful for the assessment of the global context of at least the more prominent coma structures.

Global description: in January and early February 2005 the only morphological structure noticed in the coma is a very short straight feature at position angle (PA) 225 deg that turns into tail direction (280-290 deg) at some $10000 \mathrm{~km}$ projected distance from the nucleus. As of mid February 2005 the structure in the south-western coma quadrant could be resolved into a coma fan. In early March further straight or only slightly curved features appeared in the southern coma hemisphere. In April 2005 the coma morphology evolved further, now resembling very much the porcupine pattern seen in Comet C/1995 O1 (Hale-Bopp) during 1996 (Boehnhardt et al. 1999; Sekanina \& Boehnhardt 1999): 6 features, resembling straight or moderatly curved jets, can be identified in the PA range 110-300 deg. This coma pattern continued throughout May until mid June 2005, although the straight jet-like feature $\mathrm{C}$ at $\mathrm{PA} \sim 160 \mathrm{deg}$, that was already weak in April 2005 (but detectable during 4 observing nights), could not be identified in our later exposures. As of late May 2005, jet G appeared double. The temporal evolution of the overall pattern is slow, i.e. the features changed relative intensity and orientation only slightly over months. The typical extension of the features range between a few 1000 to more than $20000 \mathrm{~km}$ projected distance from the nucleus. An additional continuous morphological structure was detected between mid March and 18 June 2005 in the northern coma hemisphere: a short, about $45 \mathrm{deg}$ wide fan (labeled A in Fig. 2) was seen at PA 340-50 deg, occasionally displaying a very sharp curvature into tail direction. Table 2 summarizes geometric information (near-nucleus 

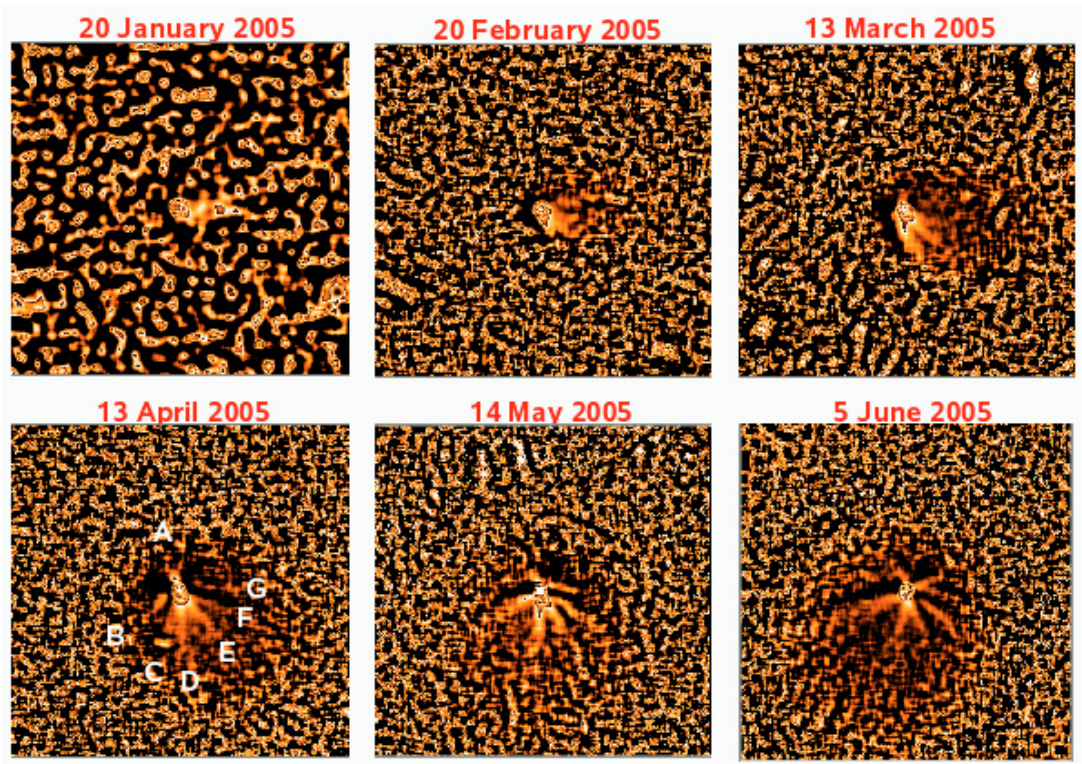

Fig. 2. Laplace filtered images of the coma structures of Comet 9P/Tempel 1. The sequence shows the Laplace filtered version of the images in Fig. 1. Laplace filtering enhances shortscale brightness gradients in the images, while at the same time suppressing the longscale ones. The irregular pattern surrounding the inner coma structures shows the noise structure in the image. The identified coma structures listed in Table 2 are labeled in the lower left sub-image. Orientation and field of view as in Fig. 1.

Table 2. Near-nucleus position angles of coma structures in 9P/Tempel 1.

\begin{tabular}{lccccccc}
\hline \hline Date & $\mathrm{A}$ & $\mathrm{B}$ & $\mathrm{C}$ & $\mathrm{D}$ & $\mathrm{E}$ & $\mathrm{F}$ & $\mathrm{G}$ \\
\hline $2005 / 01 / 20$ & ND & ND & ND & $\mathrm{MA}$ & $225 / \mathrm{c}$ & $\mathrm{ND}$ & $\mathrm{ND}$ \\
$2005 / 02 / 20$ & $\mathrm{MA}$ & $\mathrm{MA}$ & $\mathrm{ND}$ & $185 / \mathrm{c}$ & $220 / \mathrm{c}$ & $\mathrm{ND}$ & $285 / \mathrm{s} ?$ \\
$2005 / 03 / 17$ & $10 / \mathrm{s}$ & $120 / \mathrm{u}$ & $\mathrm{ND}$ & $180 / \mathrm{s}$ & $215 / \mathrm{s}$ & $240 / \mathrm{c}$ & $285 / \mathrm{s}$ \\
$2005 / 04 / 13$ & $20 / \mathrm{s}$ & $110 / \mathrm{c}$ & $165 / \mathrm{s}$ & $185 / \mathrm{s}$ & $215 / \mathrm{s}$ & $235 / \mathrm{c}$ & $290 / \mathrm{s}$ \\
$2005 / 05 / 14$ & $10 / \mathrm{c} ?$ & $115 / \mathrm{c}$ & $\mathrm{ND}$ & $180 / \mathrm{s}$ & $210 / \mathrm{s}$ & $240 / \mathrm{cc}$ & $290 / \mathrm{cc}$ \\
$2005 / 06 / 05$ & $5 / \mathrm{c}$ & $115 / \mathrm{s}$ & $\mathrm{ND}$ & $170 / \mathrm{s}$ & $205 / \mathrm{s}$ & $240 / \mathrm{cc} ?$ & $300 / \mathrm{cc} ?$ \\
$2005 / 06 / 18$ & MA & $105 / \mathrm{s}$ & $\mathrm{ND}$ & $170 / \mathrm{s}$ & $195 / \mathrm{s}$ & $\mathrm{MA}$ & $285 / \mathrm{s}$ \\
\hline
\end{tabular}

The identification of the coma structures is done by letters ABCDEF in alphabetic order for increasing position angle (see Fig. 2, lower left panel).

The given position angles refer to estimated center lines of the respective coma features. They are given to the nearest 5 deg and may have uncertainties of the same order to maximum $10 \mathrm{deg}$. North $=0 \mathrm{deg}$, East $=90 \mathrm{deg}$. The small letters after the slash indicated the curvature of the feature - for abbreviations see below.

Abbreviation: $\mathrm{MA}=$ marginal, $\mathrm{ND}=$ not detected,$/ \mathrm{cc}=$ counterclockwise curvature,$/ \mathrm{c}=$ clockwise curvature $, / \mathrm{s}=\mathrm{straight}, / \mathrm{u}=\mathrm{undetermined}$, $?=$ uncertain

position angle and curvature) of the coma structures as determined from the selected best images of our complete data set.

Another coma phenomenon of temporary nature is worthy of note: on 14 June 2005 an almost straight arclet structure extended across the western coma hemisphere between PA 225-355 deg (Fig. 3). The arclet is not detected in our comet images of 12 June 2005 or before, nor in the ones taken after 14 June 2005. It may thus be considered a temporary aftermath of dust, originating from a short outburst sometime between 12 and 14 June 2005.

Interpretation: since the contribution of light from gas and ion emissions in the $R$ and $I$ filter bands is small, if at all present before April 2005, we interpret the observed coma structures as due to dust reflected sunlight. The porcupine pattern with only minor changes of the near-nucleus position angles with time suggests the interpretation as an embedded fan coma
(Sekanina \& Boehnhardt 1999). In this scenario the emission cones produced by active regions on the nucleus, are seen more or less side-on from Earth. Thus, for the observer on Earth they appear as embedded fans projected on top of each other in the sky. The borderlines of these projected fans are the straight or curved features seen in our enhanced images (however, not every cone may produce two sharp border lines - see Sekanina 1987). The number of straight jets found in our images suggests the presence of at least 3 or 4 very active regions on the nucleus, depending on the assumed association of these jets with borderlines of coma fans. Detailed model calculations are required to link the observed coma structures with active regions on the rotating nucleus. However, a first assessment of the slow drift of position angles observed, supports two scenarios: a rotation axis orientation either (1) close to a solution found earlier from an analysis of the photometric light curve of the comet 

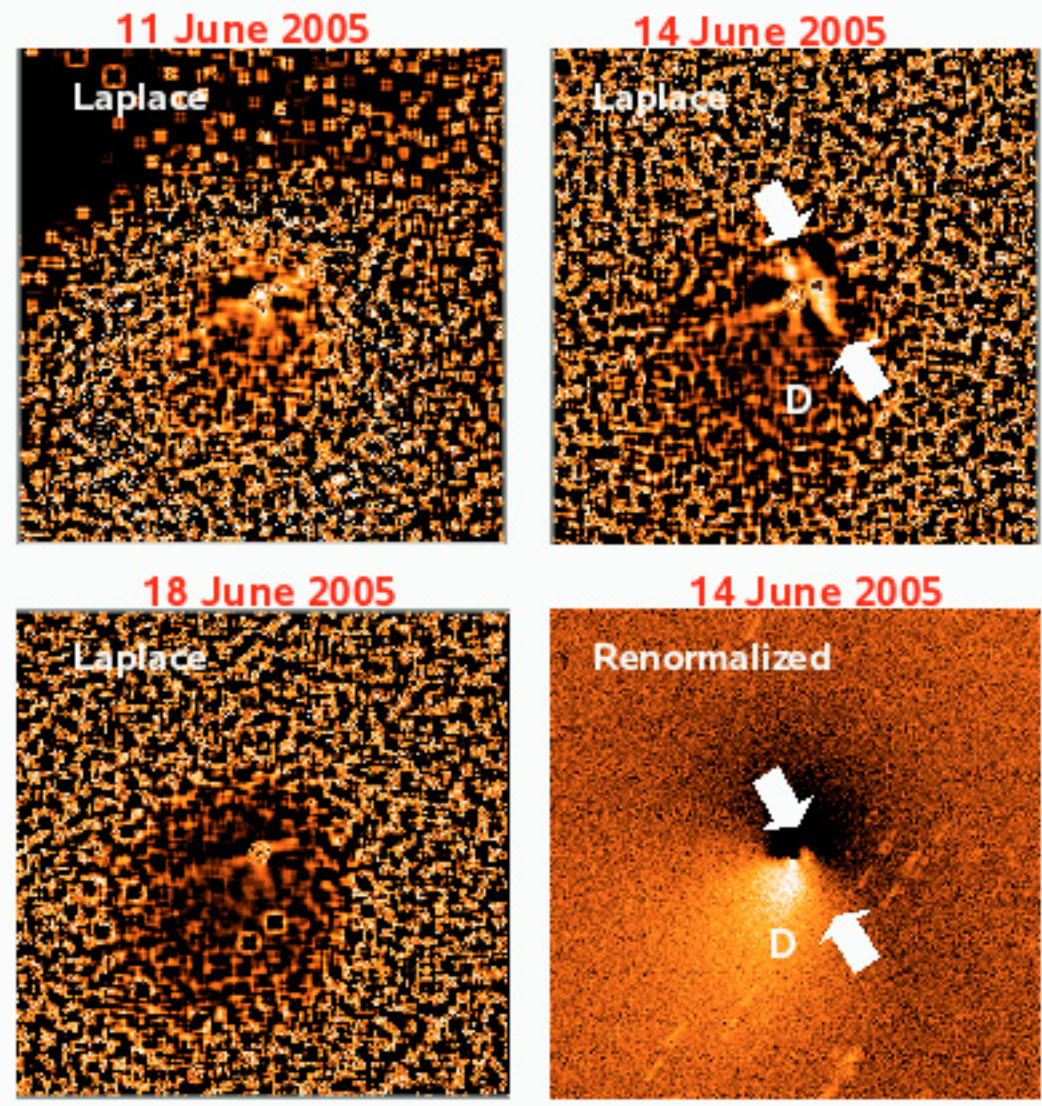

Fig. 3. The outburst on 14 June 2005. The figure shows the coma structures as seen between 11 and 18 June 2005 . On 14 June 2005 an arcletlike feature (indicated by arrows next to jet D) is visible in the western coma hemisphere. The feature is not detected in earlier (see for instance image of 11 June 2005) and later (see for instance image of 18 June 2005) exposures of the comet. The processsing technique used for coma structure enhancement is indicated in the subframes. Orientation and field of view as in Fig. 1.

(Belton et al. 2005); or (2) close to the angular momentum vector of the orbital motion of the comet. The latter scenario relies on the interpretation of jet $\mathrm{D}$ and $\mathrm{E}$ as being caused by the same active region creating a fan structure towards the south (indicated by letter D in lower left panel of Fig. 2, and in upper and lower right panel of Fig. 3).

\subsection{Af $\rho$ and spatial profiles}

The dust release is measured through optical measurements of the parameter $A(\theta) f \rho$ (A'Hearn et al. 1984) as a function of the projected cometocentric distance $\rho$. This parameter is not necessarily a good measure of the total dust production rate, but it provides an indication of small dust particles in the coma. Table 1 shows the $A f \rho$ at $\rho \sim 5000 \mathrm{~km}$. These values are applicable for $R$ Johnson, that is, when the comet was imaged with $I$ Johnson, the deduced $A f \rho$ was transformed into $R$ Johnson using colour information on the comet from the CAFOS instrument and scaled to the respective central wavelength difference of the BUSCA $I$ filter to Johnson $R$ (this assumes constant dust reddening with wavelength between the various filter bands).

The $A f \rho$ value varies with heliocentric distance as $r_{\mathrm{h}}^{-6.71}$ between Jan. 5 and Apr. 18, 2005; slightly enhanced $A f \rho$ (above the $r_{\mathrm{h}}^{-6.71}$ curve) was observed from mid-February until the end of March, when fan-shaped structures appeared in the coma for the first time (see Fig. 2). Information on the $A f \rho$ parameter between mid-April to mid-May is not available as the instrument set-up on the telescope was not appropriate for doing broadband imaging. During this period of time (i.e. 80 to 60 days before perihelion) the comet peaked in dust activity. From May 11 until June 18, 9P/Tempel 1 has experienced a continuous decrease in the dust production rate with some sporadic events of increased activity.

The azimuthally averaged profiles of the comet continuum intensity versus $\rho$ and the radial profiles of surface brightness $B$ vs. $\rho$ in north-south direction from the spectra, can be linearly fit if expressed in log-log representation. This least-square fit is done within lower and upper limits for $\rho$ given by the size of the typical seeing disk ( $1200 \mathrm{~km}$ projected distance from the nucleus) and the maximum coma extension $(35000 \mathrm{~km}$ projected distance from the nucleus) with good SNR for the comet images, respectively. The values of the slopes $m$ are listed in Table 1.

The gradient $m$ of the dust coma of 9P/Tempel 1 shows an increase from -1.9 and -1.7 in January 2005 to -1.15 and -1.2 in June 2005 as clearly seen in Fig. 4. Let us note that the values of $m$ do not depend on sky conditions, and the same trend is observed under non-photometric conditions. The $m$ value of the Tempel 1 coma is close to -1 in June 2005, a value that is characteristic for a "steady-state" coma of homogeneous 


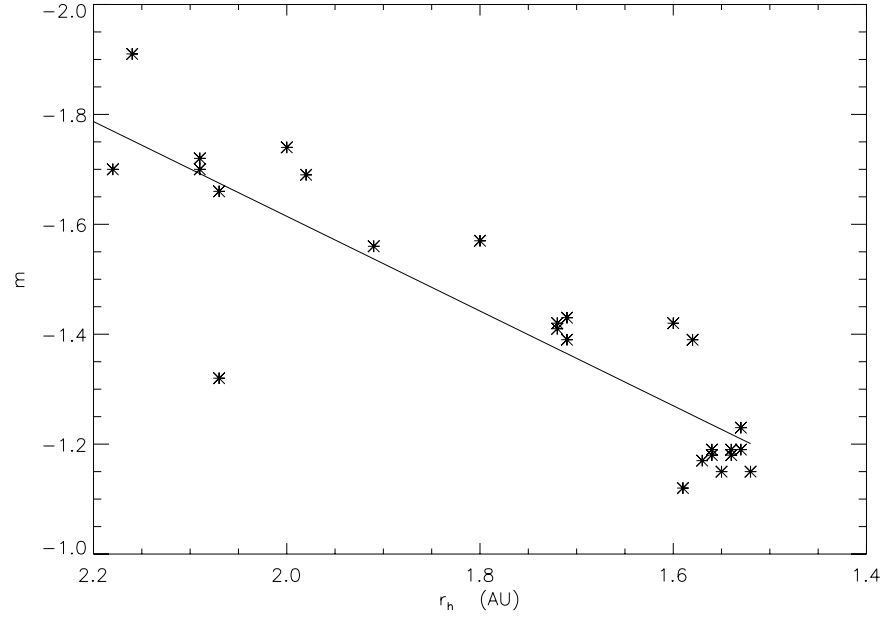

Fig. 4. Values of the slope derived from linear fits of radial profiles of continuum surface brightness vs. projected cometocentric distance $\rho$ at distances $3.1 \leq \log \rho \leq 4.5$, i.e. $\rho \sim 1200 \mathrm{~km}$ and $\rho \sim 35000 \mathrm{~km}$ at the comet distance. Standard deviation of these fits is always $\leq 1 \%$. Solid line, representing a linear fit of $m\left(r_{\mathrm{h}}\right)$ vs. $r_{\mathrm{h}}$ (standard deviation $\sim 8 \%$ ) is included as merely indicative of such a behaviour.

outflow of the dust. However, the dust coma of the comet clearly deviates from this case during the phase of early activity further away from the Sun, suggesting that the grain size distribution changes considerably with distance from the nucleus. Potentially, such changes in the grain size distribution could be due to a more stringent radiation pressure sorting of the grains throughout the coma when the comet is farther away from the Sun (larger and heavier grains stay much longer in the coma than lighter and smaller ones) or due to changes of the grain size distribution due to sublimation and/or fragmentation phenomena in the coma. Discriminative and quantitative interpretation of our measurements will require careful modelling of these effects beyond the scope of this paper.

The spectra of the comet are used to investigate the $\mathrm{CN}, \mathrm{C}_{2}$, and $\mathrm{C}_{3}$, and dust continuum profiles in the north-south direction. Let us note that no ion features have been found in any of the acquired spectra from mid-April to mid-June. The spectral regions of the clearly detected gas emissions and the subtraction of the underlying continuum in the gas emission bands is done as described by Lara et al. (2001). The conversion of the emission band fluxes into column densities makes use of constant $g$-factors for $\mathrm{C}_{2}$ and $\mathrm{C}_{3}$ (A'Hearn et al. 1995), whereas the $g$-factor of the $\mathrm{CN}$ molecule is calculated for the heliocentric distance and velocity of 9P/Tempel 1 on every date from the set of values given by (Schleicher 1983). The gas production rates are obtained by means of the Haser (1957) modeling with customary $v_{p}=v_{d}=1 \mathrm{~km} \mathrm{~s}^{-1}$ and scale lengths given in A'Hearn et al. 1995. The production rates obtained and log $\left[Q\left(\mathrm{C}_{2}\right) / Q(\mathrm{CN})\right], \log \left[Q\left(\mathrm{C}_{3}\right) / Q(\mathrm{CN})\right]$ and $\log [A f \rho / Q(\mathrm{CN})]$ are listed in Table 1. On January 31, 2005, no clear gas emission is detected. On March 27, sky conditions were not photometric and $\mathrm{S} / \mathrm{N}$ was very low due to passing clouds while exposing, hampering our effort of, at least, determining quotients of gas emissions. During the visitor observing run from the 10th to the 14th of April, there were only 3 photometric nights: April 12, 13 and 14. Data acquired on those dates clearly show the $\mathrm{CN}, \mathrm{C}_{2}(\Delta v=-1)$ and $\mathrm{C}_{3}$ spectroscopic features. Their production rates could be determined and are listed in Table 1. During these 3 consecutive days, the production rate of these species experienced a slow increase, more pronounced for $\mathrm{C}_{2}$, as the comet was approaching the Sun. Further gas production rates were derived from spectra acquired on May 11 and June 18. On May 11 , the $\mathrm{CN}, \mathrm{C}_{2}$ and $\mathrm{C}_{3}$ production rates are the largest ones measured in this 6 months pre-impact monitoring, whereas measurements on June 18 seem to indicate that the gas activity started to decrease sometime after May 11, although it cannot be firmly stated given the uncertainty $(\leq 25 \%)$ in the gas production rates we have obtained. For every date, the spatial profiles of the different gas species detected are considerably asymmetric in the North-South direction as projected on the sky plane, where the slit was positioned, with the column density being higher in the South direction. It is important to note that in that direction, stable coma structures have existed as of mid February to the end of the observing interval described here. This asymmetry is more pronounced for $\mathrm{CN}$ than for any other species on every date.

Abundance ratios of $\mathrm{C}_{2}$ to $\mathrm{CN}, \mathrm{C}_{3}$ to $\mathrm{CN}$ and $\frac{A f \rho}{Q(\mathrm{CN})}$ remain relatively stable, within error bars, as the comet moved inbound. According to the taxonomical classification by A'Hearn et al. (1995) and referring to the quotients of $\mathrm{C}_{2}$ to $\mathrm{CN}$ listed in Table 1 , comet 9P/Tempel 1 can be considered as typical in terms of $\mathrm{C}_{2}$ abundance during the 2005 passage in its preperihelion phase. This result is in agreement with what Cochran (1992) found (i.e. from data acquired in 1983,9P/Tempel 1 had abundances of carbon-chain radicals relative to $\mathrm{CN}$ very near the mean values for all comets) and with A'Hearn et al. (1995) results who report a value of $\log \frac{Q\left(\mathrm{C}_{2}\right)}{Q(\mathrm{CN})} \sim-0.09$ to be compared with $0.05 \pm 0.08$ as a mean value of the ones listed in Table 1 . Note that Table VI in A'Hearn et al. (1995) gives a mean value of $\log \frac{Q\left(\mathrm{C}_{2}\right)}{Q(\mathrm{CN})}=0.06 \pm 0.10$ for typical comets whereas the full range is -0.09 to 0.29 .

Comparison of our pre-perihelion results with production rates from the 1983 apparition (Osip et al. 1992; Storrs et al. 1992) seems to point to a lower $\mathrm{CN}$ production rate during the 2005 passage. Additionally, values presented here and in Storrs et al. (1992) reproduce a decrease in $\mathrm{Q}(\mathrm{CN})$ at heliocentric distances shorter than $1.7 \mathrm{AU}$, contrary to Osip et al. (1992) where relatively constant $\mathrm{CN}$ production rates can be found at $r_{\mathrm{h}} \leq 1.7 \mathrm{AU}$. Regarding the parameter $A f \rho$, its varition with $r_{\mathrm{h}}$ does not seem to have gone through clear changes from previous passages to this one, the peak in the production of small dust particles occurs somewhere between 1.74 and 1.60 AU heliocentric distance. In overall, the Af $\rho$ values at $r_{\mathrm{h}}=2.21-1.51 \mathrm{AU}$ during the 2005 apparition are systematically below those presented in Osip et al. (1992) and Storrs et al. (1992), which pertain to the 1983 passage.

\section{Conclusions}

We have monitored the comet 9P/Tempel 1 from January until June, 2005, aiming at a characterization of the comet behaviour as approaching to the perihelion, the time at which the Deep Impact spacecraft has successfully delivered a projectile to 
impact the nucleus. The limited mission payload and the short time the comet was observed before and after the impact by the cameras onboard the spacecraft is making the coordinated ground-based observations the most important means to unveil the nature of these primitive bodies of the Solar System.

The monitoring followed up at Calar Alto is unique in this sense, as images and spectra of the comet have been acquired in a regular base for six months. From this data, we have determined the evolution of the gas production rates, of the gasto-dust ratios and of the relative abundance of different gases $\left(\mathrm{CN}, \mathrm{C}_{2}, \mathrm{C}_{3}\right)$, and we have obtained firm indications of the rotation state and the distribution of active areas of the nucleus. Hence, this has allowed us to establish (i) pre-impact activity baselines and (ii) the likely rotational state of the nucleus. Both results are crucial for a full understanding of the overall effects the impact has produced on the comet nucleus.

Furthermore, from comet splitting events (when small natural craters are formed at the nuclear surface) a time scale of 1-5 days is known in which changes in the cometary activity evolve (Boehnhardt 2004). Due to this, our monitoring of 9P/Tempel 1 continued until July 14 to observe the evolution of the gas and dust production rates. In this follow up, recently finished, we also aim to detect the existence of new coma structures, if they appear as an aftermath of the impact, as activity indicators and from that to establish a map of active regions on the nucleus, in order to be compared with those obtained in the pre-impact phase. The results derived from data acquired after June 18 and until July 14 will be analysed, interpreted and presented in a forthcoming paper.

Acknowledgements. Based on observations collected at the Centro Astronómico Hispano Alemán (CAHA) at Calar Alto, operated jointly by the Max-Planck Institut für Astronomie and the Instituto de Astrofísica de Andalucía (CSIC), and at the Sierra Nevada Observatory operated by the Instituto de Astrofísica de Andalucía
(CSIC). We are grateful to the service observers J. Aceituno, A. Aguirre, M. Alises, N. Cardiel, V. Casanova, A. Guijarro, F. Hoyo, S. Pedraz, S. Sánchez, A. Sota, U. Thiele at both observatories without their engagement and dedication this program would not have been successful.

The research carried out has been partially supported by the Spanish Ministerio de Ciencia y Tecnología under contracts ESP2003-0357 and PNAYA-2002-0382.

\section{References}

A'Hearn, M. F., Schleicher, D. G., Millis, R. L., et al. 1984, AJ, 89, 579.

A'Hearn M. F., Hoban S., Birch, P. V., et al. 1986, Nature, 324, 649

A’Hearn, M. F., Millis, R. L. Schleicher, et al., 1995, Icarus, 118, 223

Belton, M. S., Meech, K. J., A'Hearn, M. F., et al., 2005, Space Sci. Rev., 117, 137

Boehnhardt, H., \& Birkle, K. 1994, A\&AS, 107, 101

Boehnhardt, H., Birkle, K., Fiedler, A., et al., 1999, EMP, 78, 179

Boehnhardt, H. 2004, in Comets II, ed. M. Festou, H. U. Keller, \& H. Weaver (University of Arizona Press), 301

Cochran, A. L., Barker, E. S., Ramseyer, T. F., \& Storrs A. D. 1992, Icarus, 98,151

Haser, L., 1957, Bull. Cl. Sci. Acad. R. Belg., 43, 740.

Lara, L. M., Schulz, R., Stuewe, J. A., \& Tozzi, G. P. 2001, Icarus, 150, 124.

Lara, L. M., Tozzi, G. P., Boehnhardt, H., DiMartino, M., \& Schulz, R. 2004a, A\&A 422, 717

Lara, L. M., Rodrigo, R., Tozzi, G. P, Boehnhardt, H., \& Leisy, P. 2004b, A\&A 423, 1169

Meech, K. J., A'Hearn, M. F., Fernandez, Y. R., et al. 2005, Space Sci. Rev., 117, 297

Osip, D. J., Schleicher, D. G., \& Millis, R. L. 1992, Icarus, 98, 115

Schleicher, D. G. 1983, Ph.D. Dissertation, Univ. of Maryland

Sekanina, Z. 1987, ESA SP-278, 315

Sekanina, Z., \& Boehnhardt, H. 1999, EMP, 78, 313

Storrs, A. D., Cochran, A. L., \& Barker, E. S. 1992, Icarus, 98, 163 